\title{
Status of natural supersymmetry from the generalized minimal supergravity in light of the current LHC run-2 and LUX data
}

\author{
Waqas Ahmed, ${ }^{1,3}$ Xiao-Jun Bi, ${ }^{2,3}$ Tianjun Li, ${ }^{1,3}$ Jia-Shu Niu, ${ }^{1,3}$ Shabbar Raza, ${ }^{4}$ Qian-Fei Xiang, ${ }^{2,3}$ and Peng-Fei Yin ${ }^{2}$ \\ ${ }^{1}$ CAS Key Laboratory of Theoretical Physics, Institute of Theoretical Physics, \\ Chinese Academy of Sciences, Beijing 100190, China \\ ${ }^{2}$ Key Laboratory of Particle Astrophysics, Institute of High Energy Physics, Chinese Academy of Sciences, \\ Beijing 100049, China \\ ${ }^{3}$ School of Physical Sciences, University of Chinese Academy of Sciences, \\ No. 19A Yuquan Road, Beijing 100049, China \\ ${ }^{4}$ Department of Physics, Federal Urdu University of Arts, Science and Technology, \\ Karachi 75300, Pakistan
}

(Received 30 October 2017; published 31 July 2018)

\begin{abstract}
We study natural supersymmetry in the generalized minimal supergravity (GmSUGRA). For the parameter space with low energy, electroweak fine-tuning measures less than 50, we are left with only the Z-pole, Higgs-pole, and Higgsino LSP scenarios for dark matter (DM). We perform the focused scans for such parameter space and find that it satisfies various phenomenological constraints and is compatible with the current direct detection bound on neutralino DM reported by the LUX experiment. Such parameter space also has solutions with correct DM relic density besides the solutions with DM relic density smaller or larger than $5 \sigma$ WMAP9 bounds. We present five benchmark points as examples. In these benchmark points, the gluino and the first two generations of squarks are heavier than $2 \mathrm{TeV}$, stop $\tilde{t}_{1,2}$ are in the mass range $[1,2] \mathrm{TeV}$, while sleptons are lighter than $1 \mathrm{TeV}$. Some part of the parameter space can explain the muon anomalous magnetic moment within $3 \sigma$ as well. We also perform the collider study of such solutions by implementing and comparing with relevant studies done by the ATLAS and CMS Collaborations. We find that the points with Higgsino dominant $\tilde{\chi}_{2}^{0} / \tilde{\chi}_{1}^{ \pm}$mass up to $300 \mathrm{GeV}$ are excluded in $Z$-pole scenario while for Higgs-pole scenario, the points with $\tilde{\chi}_{2}^{0}$ mass up to $460 \mathrm{GeV}$ are excluded. We also notice that the Higgsino LSP points in our present scans are beyond the reach of present LHC searches. Next, we show that for both the $Z$-pole and Higgs-pole scenarios, the points with electroweak fine-tuning measures around 20 do still survive.
\end{abstract}

DOI: $10.1103 /$ PhysRevD.98.015040

\section{INTRODUCTION}

Undoubtedly, the gauge coupling unification of the strong, weak and electromagnetic interactions of the fundamental particles is a great triumph of the supersymmetric (SUSY) version of the standard model (SM) of particle physics [1], which henceforth will be called the supersymetric SM (SSM). The SSM predicts the existence of SUSY partners of all the known SM particles. Interestingly, the existence of these particles can help us to understand the stabilization of the electroweak (EW) scale and thus solves yet another daunting problem of particle physics named as the gauge hierarchy problem [2]. In addition, the minimal

Published by the American Physical Society under the terms of the Creative Commons Attribution 4.0 International license. Further distribution of this work must maintain attribution to the author(s) and the published article's title, journal citation, and DOI. Funded by SCOAP .
SSM (MSSM) also predicts the Higgs boson mass $\left(m_{h}\right)$ should be smaller than $135 \mathrm{GeV}$ [3]. Indeed, the ATLAS and CMS Collaborations of the Large Hadron Collider (LHC) have discovered a SM-like Higgs boson $h$ with mass $m_{h}=$ $125 \mathrm{GeV}[4,5]$. This adds yet another feather in the hat of the SSM. The SSM also predicts that with $R$-parity conservation, the lightest supersymmetric particle (LSP) such as the neutralino is an excellent dark matter candidate [6,7]. And the electroweak symmetry can be broken radiatively due to the large top quark Yukawa coupling, etc. All these observations give us some hints that we are on the right track.

The existence of the SM-like Higgs boson with mass $m_{h} \sim 125 \mathrm{GeV}$ requires the multi-TeV top squarks with small mixing or $\mathrm{TeV}$-scale top squarks with large mixing. This raises a question on the naturalness of the MSSM and generates the fine-tuning problem. However, the null results of the LHC-Run2 and the ongoing LHC SUSY-searches have not found any SUSY evidences yet. In recent studies, the bounds on squark masses $m_{\tilde{q}} \gtrsim 1600 \mathrm{GeV}$ [8] and 
gluino mass $m_{\tilde{q}} \gtrsim 2000 \mathrm{GeV}$ [8] have been reported by the ATLAS and CMS Collaborations at the $13 \mathrm{TeV}$ LHC with $36 \mathrm{fb}^{-1}$ of data. This situation has put the promises of the MSSM under pressure. It is interesting to note that despite the SM-like Higgs mass being relatively heavy, there are some studies [9-21] which suggest that the naturalness problem in the MSSM can be solved successfully. In particular, in an interesting scenario, which is called the supernatural SUSY [17,22], it can be shown that no residual electroweak fine-tuning (EWFT) left in the MSSM if we employ the No-Scale supergravity boundary conditions [23] and Giudice-Masiero (GM) mechanism [24] despite having relatively heavy spectra. Some people might think that the supernatural SUSY might have a problem related to the Higgsino mass parameter $\mu$, which is generated by the GM mechanism and is proportional to the universal gaugino mass $M_{1 / 2}$, since the ratio $M_{1 / 2} / \mu$ is of order one but cannot be determined as an exact number. This problem, if it is, can be addressed in the M-theory inspired the Next to MSSM (NMSSM) [25]. Also, see [26], for more recent works related to naturalness within and beyond the MSSM.

In order to quantify the amount of fine-tuning (FT), we need to define the fine-tuning measures. In the literature, we can find the high-energy fine-tuning measure $\Delta_{\text {EENZ-BG }}$ defined by Ellis, Enqvist, Nanopoulos, and Zwirner [27], as well as Barbieri and Giudice [28], and the high-energy and electroweak fine-tuning measures $\Delta_{\mathrm{HS}}$ and $\Delta_{\mathrm{EW}}$ defined by Baer, Barger, Huang, Michelson, Mustafayev, and Tata [29-31]. Usually, we have $\Delta_{\mathrm{EW}} \lesssim \Delta_{\mathrm{BG}} \lesssim \Delta_{\mathrm{HS}}$. One can show that $\Delta_{\mathrm{EW}} \sim \Delta_{\mathrm{BG}}$ for some scenarios [32].

This work is a continuation of our phenomenological studies of the generalized minimal supergravity model (GmSUGRA) [33]. In Refs. [34,35], we showed that in GmSUGRA, we have varieties of dark matter scenarios such as $A$-resonance, Higgs-resonance, $Z$-resonance, stau-neutralino coannihilation, tau sneutrino-neutralino coannihilation compatible with various phenomenological constraints. In addition, we showed that the Higgs coupling and muon anomalous magnetic moment measurements can constrain the parameter space effectively. In this work, we concentrate on the dark matter solutions which not only have low EWFT (that is $\Delta_{\mathrm{EW}} \lesssim 50$ ), but also are consistent with current direct detection bounds reported by the LUX Collaboration [36]. In our scans, we find that the light stau-neutralino coannihilation points do not satisfy $\Delta_{\mathrm{EW}} \lesssim 50$. Also, the Higgsino LSP points are still natural and viable, but they cannot be probed at the current LHC searches. We find that only Higgs-pole and Z-pole solutions fulfill the above-mentioned criteria. Therefore, we will only consider these two types of resonance points in more detail. In these two scenarios, a subset of solutions satisfies the $5 \sigma$ dark matter relic density WMAP9 bounds, while the other solutions have relic density beyond the $5 \sigma$ bounds. We present five benchmark points as examples of the parameter space under consideration, where one of them has the
Higgsino LSP. In these benchmark points, gluino and the first two generations of squarks are heavier than $2 \mathrm{TeV}$, top squarks $\tilde{t}_{1,2}$ are in the mass range $[1,2] \mathrm{GeV}$, while sleptons are less than $1 \mathrm{TeV}$. Some part of the parameter space can also explain the muon anomalous magnetic moment within $3 \sigma$ [37]. Furthermore, we consider the constraints on such solutions from the direct searches for the SUSY particles at the LHC. In order to realize small fine-tuning and satisfy experimental constraints simultaneously, only electroweakinos (neutralinos and charginos) and stau are light and could be explored at the current LHC searches. We study various electroweak Drell-Yan production processes where one could produce neutralinos which could decay through on-shell or off-shell $Z^{(*)}\left(\tilde{\chi}_{i}^{0} \rightarrow Z^{(*)} \tilde{\chi}_{1}^{0}\right)$ or $h^{(*)}\left(\tilde{\chi}_{i}^{0} \rightarrow h^{(*)} \tilde{\chi}_{1}^{0}\right)$. We will give more details about our analyses later in this paper. We display various plots showing that the relevance of different decay modes depends on mass spectra and will significantly influence collider searches for these particles. The dominant decay channel of $\tilde{\chi}_{2}^{0}$ for samples of $Z$-pole is $\tilde{\chi}_{2}^{0} \rightarrow \tilde{\chi}_{1}^{0} Z^{(*)}$ when the mass difference $m_{\tilde{\chi}_{2}^{0}}-m_{\tilde{\chi}_{1}^{0}}$ is small. Once the decay into the Higgs boson is kinematically possible, the branching ratio to $\tilde{\chi}_{1}^{0} h$ increases with increasing of $m_{\tilde{\chi}_{2}^{0}}-m_{\tilde{\chi}_{1}^{0}}$ and becomes the dominant channel when $m_{\tilde{\chi}_{2}^{0}}-m_{\tilde{\chi}_{1}^{0}} \gtrsim 140 \mathrm{GeV}$. The decay channels of $\tilde{\chi}_{1}^{ \pm}$is always $\tilde{\chi}_{1}^{ \pm} \rightarrow \tilde{\chi}_{1}^{0} W^{(*)}$. We also find that, for our present work, $3 l+E_{\mathrm{T}}$ and $2 l+E_{\mathrm{T}}$ give the best sensitivity at the LHC searches where electroweakinos decay to multileptons. We use suitable kinematic variables to discriminate signals from backgrounds. We show the $95 \%$ C.L. exclusion results of the LHC electroweakinos searches in the $m_{\tilde{\chi}_{1}^{0}}-m_{\tilde{\chi}_{2}^{0}}$ plane and $m_{\tilde{\chi}_{1}^{0}}-\Delta_{\text {EW }}$ plane. It can be seen from these plots that Higgsino dominant $\tilde{\chi}_{2}^{0} / \tilde{\chi}_{1}^{ \pm}$with mass up to $300 \mathrm{GeV}$ are excluded in case of $Z$-pole while for Higgs-pole scenario, points with $\tilde{\chi}_{2}^{0}$ mass up to $460 \mathrm{GeV}$ are excluded. Moreover, it can also be noticed that $Z$-pole solutions with small $\Delta_{\mathrm{EW}}$ are easy to be explored, whereas solutions with large $\Delta_{\mathrm{EW}}$ are hard to exclude but for the Higgs-pole, many points with $\Delta_{\mathrm{EW}}$ up to 50 could by excluded by electroweakino searches with tau final states. Finally, we notice that for both the $Z$-pole and the Higgs-pole, samples with $\Delta_{\mathrm{EW}} \sim 20$ could still survive, indicating naturalness of this SUSY framework.

The remainder of this paper is organized as follows. We present our model in Sec. II. We discuss the EWFT measure in Sec. III. Section IV is devoted to scanning procedures and phenomenological constraints. Our results for focused scans are shown in Sec. V, while results for the LHC searches are presented in Sec. VI. A summary and conclusion are given in Sec. VII.

\section{THE ELECTROWEAK SUSY FROM THE GmSUGRA IN THE MSSM}

In GmSUGRA, at the GUT-scale, we can write the generalized gauge coupling relation and the generalized gaugino mass relation as follows, 


$$
\begin{gathered}
\frac{1}{\alpha_{2}}-\frac{1}{\alpha_{3}}=k\left(\frac{1}{\alpha_{1}}-\frac{1}{\alpha_{3}}\right), \\
\frac{M_{2}}{\alpha_{2}}-\frac{M_{3}}{\alpha_{3}}=k\left(\frac{M_{1}}{\alpha_{1}}-\frac{M_{3}}{\alpha_{3}}\right),
\end{gathered}
$$

where $k$ is the index of these relations since it is invariant under the one-loop renormalization group equation (RGE) running. For more details about the model, please see [33].

Another important feature of GmSUGRA is that we can realize Electroweak SUSY (EWSUSY). In this scenario, we can have the sleptons and electroweakinos within one $\mathrm{TeV}$ while squarks and/or gluinos can be in several $\mathrm{TeV}$ mass ranges [38]. Assuming gauge coupling unification at the GUT scale $\left(\alpha_{1}=\alpha_{2}=\alpha_{3}\right)$ and using $k=5 / 3$, we obtain a simple gaugino mass relation from Eq. (2)

$$
M_{2}-M_{3}=\frac{5}{3}\left(M_{1}-M_{3}\right) .
$$

It is straightforward to notice that the universal gaugino mass relation $M_{1}=M_{2}=M_{3}$ in the mSUGRA, is just a special case of this general one. This is why we call it Generalized mSUGRA. We will choose $M_{1}$ and $M_{2}$ to be free input parameters, which vary around several hundred $\mathrm{GeV}$ for the EWSUSY. We can now write Eq. (3) for $M_{3}$ as

$$
M_{3}=\frac{5}{2} M_{1}-\frac{3}{2} M_{2},
$$

which could be as large as several $\mathrm{TeV}$ or as small as several hundred $\mathrm{GeV}$, depending on specific values of $M_{1}$ and $M_{2}$.

The general SUSY breaking (SSB) soft scalar masses at the GUT scale are given in Ref. [39]. Taking the slepton masses as free parameters, we obtain the following squark masses in the $S U(5)$ model with an adjoint Higgs field,

$$
\begin{aligned}
& m_{\tilde{Q}_{i}}^{2}=\frac{5}{6}\left(m_{0}^{U}\right)^{2}+\frac{1}{6} m_{\tilde{E}_{i}^{c}}^{2}, \\
& m_{\tilde{U}_{i}^{c}}^{2}=\frac{5}{3}\left(m_{0}^{U}\right)^{2}-\frac{2}{3} m_{\tilde{E}_{i}^{c}}^{2}, \\
& m_{\tilde{D}_{i}^{c}}^{2}=\frac{5}{3}\left(m_{0}^{U}\right)^{2}-\frac{2}{3} m_{\tilde{L}_{i}}^{2},
\end{aligned}
$$

where $m_{\tilde{Q}}, m_{\tilde{U}^{c}}, m_{\tilde{D}^{c}}, m_{\tilde{L}}$, and $m_{\tilde{E}^{c}}$ represent the scalar masses of the left-handed squark doublets, right-handed uptype squarks, right-handed down-type squarks, left-handed sleptons, and right-handed sleptons, respectively, while $m_{0}^{U}$ is the universal scalar mass, as in the mSUGRA. In the EWSUSY, $m_{\tilde{L}}$ and $m_{\tilde{E}^{c}}$ are both within $1 \mathrm{TeV}$, resulting in light sleptons. Especially, in the limit $m_{0}^{U} \gg m_{\tilde{L} / \tilde{E}^{c}}$, we have the approximated relations for squark masses: $2 m_{\tilde{Q}}^{2} \sim m_{\tilde{U}^{c}}^{2} \sim m_{\tilde{D}^{c}}^{2}$. In addition, the Higgs soft masses
$m_{\tilde{H}_{u}}$ and $m_{\tilde{H}_{d}}$, and the trilinear soft terms $A_{U}, A_{D}$ and $A_{E}$ can all be free parameters from the GmSUGRA [38,39].

\section{THE ELECTROWEAK FINE-TUNING}

As we mentioned earlier that in this work we are interested in solutions with low EWFT. We use the (7.85) version of ISAJET [40] to calculate the FT conditions at the EW scale $M_{\mathrm{EW}}$. After including the one-loop effective potential contributions to the tree-level MSSM Higgs potential, the $Z$-boson mass $M_{Z}$ is given by

$$
\frac{M_{Z}^{2}}{2}=\frac{\left(m_{H_{d}}^{2}+\Sigma_{d}^{d}\right)-\left(m_{H_{u}}^{2}+\Sigma_{u}^{u}\right) \tan ^{2} \beta}{\tan ^{2} \beta-1}-\mu^{2},
$$

where $\Sigma_{u}^{u}$ and $\Sigma_{d}^{d}$ are the contributions coming from the one-loop effective potential defined in Ref. [30] and $\tan \beta \equiv \frac{v_{u}}{v_{d}}$. All parameters in Eq. (8) are defined at the $M_{\mathrm{EW}}$. In order to measure the EWFT condition, we follow [30] and use the following definitions,

$C_{H_{d}} \equiv\left|m_{H_{d}}^{2} /\left(\tan ^{2} \beta-1\right)\right|$,

$C_{H_{u}} \equiv\left|-m_{H_{u}}^{2} \tan ^{2} \beta /\left(\tan ^{2} \beta-1\right)\right|, \quad C_{\mu} \equiv\left|-\mu^{2}\right|$,

with each $C_{\sum_{u, d}^{u, d}(r)}$ less than some characteristic value of order $M_{Z}^{2}$. Here, $r$ labels the SM and SUSY particles that contribute to the one-loop Higgs potential. For the finetuning measure, we define

$$
\Delta_{\mathrm{EW}} \equiv \max \left(C_{r}\right) /\left(M_{Z}^{2} / 2\right) .
$$

Note that $\Delta_{\mathrm{EW}}$ only depends on the weak-scale parameters of the SSMs, and then is fixed by the particle spectra. Hence, it is independent of how the SUSY particle masses arise. Lower values of $\Delta_{\mathrm{EW}}$ corresponds to less fine tuning, for example, $\Delta_{\mathrm{EW}}=50$ implies $\Delta_{\mathrm{EW}}^{-1}=2 \%$ fine tuning. In addition to $\Delta_{\mathrm{EW}}$, ISAJET also calculates $\Delta_{\mathrm{HS}}$ which is a measure of fine-tuning at the high scale (HS) like the GUT scale in our model [30]. The HS fine-tuning measure $\Delta_{\mathrm{HS}}$ is given as follows:

$$
\Delta_{\mathrm{HS}} \equiv \max \left(B_{i}\right) /\left(M_{Z}^{2} / 2\right) .
$$

For definition of $B_{i}$ and more details, please see Ref. [30].

\section{SCANNING PROCEDURE AND PHENOMENOLOGICAL CONSTRAINTS}

We employ the ISAJET 7.85 package [40] to perform the focused scans using parameters given in Sec. II to explore the parameter space having Z-resonance and Higgsresonance solutions. In this work, we will focus on the solutions with relatively small EWFT $\Delta_{\mathrm{EW}} \lesssim 50$. For full ranges of the parameter see [34]. 
In ISAJET, the weak scale values of the gauge and third generation Yukawa couplings are evolved to $M_{\mathrm{GUT}}$ via the MSSM renormalization group equations (RGEs) in the $\overline{\mathrm{DR}}$ regularization scheme. We do not strictly enforce the unification condition $g_{3}=g_{1}=g_{2}$ at $M_{\mathrm{GUT}}$, since a few percent deviation from unification can be assigned to the unknown GUT-scale threshold corrections [41]. With the boundary conditions given at $M_{\mathrm{GUT}}$, all the SSB parameters, along with the gauge and Yukawa couplings, are evolved back to the weak scale $M_{\mathrm{Z}}$.

In evaluating Yukawa couplings, the SUSY threshold corrections [42] are taken into account at the common scale $M_{\mathrm{SUSY}}=\sqrt{m_{\tilde{t}_{L}} m_{\tilde{t}_{R}}}$. The entire parameter set is iteratively run between $M_{\mathrm{Z}}$ and $M_{\mathrm{GUT}}$ using the full two-loop RGEs until a stable solution is obtained. To better account for the leading-log corrections, one-loop step-beta functions are adopted for gauge and Yukawa couplings, and the SSB parameters $m_{i}$ are extracted from RGEs at appropriate scales $m_{i}=m_{i}\left(m_{i}\right)$. The RGE-improved one-loop effective potential is minimized at an optimized scale $M_{\mathrm{SUSY}}$, which effectively accounts for the leading two-loop corrections. The full one-loop radiative corrections are incorporated for all sparticles.

It should be noted that the requirement of radiative electroweak symmetry breaking (REWSB) [43] puts an important theoretical constraint on parameter space. Another important constraint comes from limits on the cosmological abundance of stable charged particle [44]. This excludes regions in the parameter space where charged SUSY particles, such as $\tilde{\tau}_{1}$ or $\tilde{t}_{1}$, become the LSP. We accept only those solutions for which one of the neutralinos is the LSP.

Also, we consider $\mu>0$ and use $m_{t}=173.3 \mathrm{GeV}$ [45]. Note that our results are not too sensitive to one or two sigma variations in the value of $m_{t}$ [46]. We use $m_{b}^{\overline{\mathrm{DR}}}\left(M_{\mathrm{Z}}\right)=2.83 \mathrm{GeV}$ as well which is hard-coded into ISAJET. Also, we will use the notations $A_{t}, A_{b}, A_{\tau}$ for $A_{U}$, $A_{D}$, and $A_{E}$, respectively.

In scanning the parameter space, we employ the Metropolis-Hastings algorithm as described in [47]. The data points collected all satisfy the requirement of REWSB, with the neutralino being the LSP. After collecting the data, we require the following bounds (inspired by the LEP2 experiment) on sparticle masses.

(1) LEP2 constraints

We employ the LEP2 bounds on sparticle masses

$$
\begin{array}{r}
m_{\tilde{\tau}_{1}}, m_{\tilde{b}_{1}} \geq 100 \mathrm{GeV}, \\
m_{\tilde{\tau}_{1}} \geq 105 \mathrm{GeV}, \\
m_{\tilde{\chi}_{1}^{ \pm}} \geq 103 \mathrm{GeV} .
\end{array}
$$

(2) Higgs mass constraints

The combined value of Higgs mass reported by the ATLAS and CMS Collaborations is [48] $m_{h}=125.09 \pm 0.21$ (stat) \pm 0.11 (syst) GeV

Due to the theoretical uncertainty of $3-5 \mathrm{GeV}$ as mentioned in Ref. [49], we take the following range for Higgs boson mass:

$$
122 \mathrm{GeV} \leq m_{h} \leq 128 \mathrm{GeV} .
$$

Moreover, an approximate error of around $2 \mathrm{GeV}$ is expected in the estimation of the Higgs boson mass in Isajet which largely arises from theoretical uncertainties in the calculation of the minimum of the scalar potential, and to a lesser extent from experimental uncertainties in the values for $m_{t}$ and $\alpha_{s}$ (please see the footnote on page 10 of Ref. [50]). As a recent example, please see Ref. [51], where the uncertainty $\pm 3 \mathrm{GeV}$ was considered as well.

(3) LHC constraints

We demand [8]

$$
m_{\tilde{q}} \geq 2000 \mathrm{GeV}, \quad m_{\tilde{g}} \geq 2000 \mathrm{GeV} .
$$

(4) B-physics constraints

We use the IsAToOLs package $[52,53]$ and implement the following B-physics constraints [54,55]:

$1.6 \times 10^{-9} \leq \mathrm{BR}\left(B_{s} \rightarrow \mu^{+} \mu^{-}\right) \leq 4.2 \times 10^{-9}(2 \sigma)$,

$$
2.99 \times 10^{-4} \leq \mathrm{BR}(b \rightarrow s \gamma) \leq 3.87 \times 10^{-4}(2 \sigma),
$$

$$
0.70 \times 10^{-4} \leq \mathrm{BR}\left(B_{u} \rightarrow \tau \nu_{\tau}\right) \leq 1.5 \times 10^{-4}(2 \sigma) .
$$

(5) Electroweak Fine-Tuning constraint

Because we consider the natural SUSY, the following constraint on fine-tuning measure $\Delta_{\mathrm{EW}}$ is applied:

$$
\Delta_{\mathrm{EW}} \leq 50 .
$$

(6) WMAP constraint

We apply the WMAP9 bounds with $5 \sigma$ variation on DM density [56]

$$
0.0913 \leq \Omega h^{2} \leq 0.1363 \text {. }
$$

\section{RESULTS OF FOCUSED SCANS}

We present results of focused scans in Fig. 1. In the top right and left panels we display plots in $\Delta_{\mathrm{EW}}$ vs $\mu$ respectively for Z-pole and for Higgs-pole scenarios while rescaled spin-independent $\left(\xi \sigma^{\mathrm{SI}}(\chi, p)\right)$ rate vs LSP neutralino mass $m_{\tilde{\chi}_{1}^{0}}$ is shown in bottom panel. Aqua points satisfy the REWSB and LSP neutralino conditions. Red, blue and 

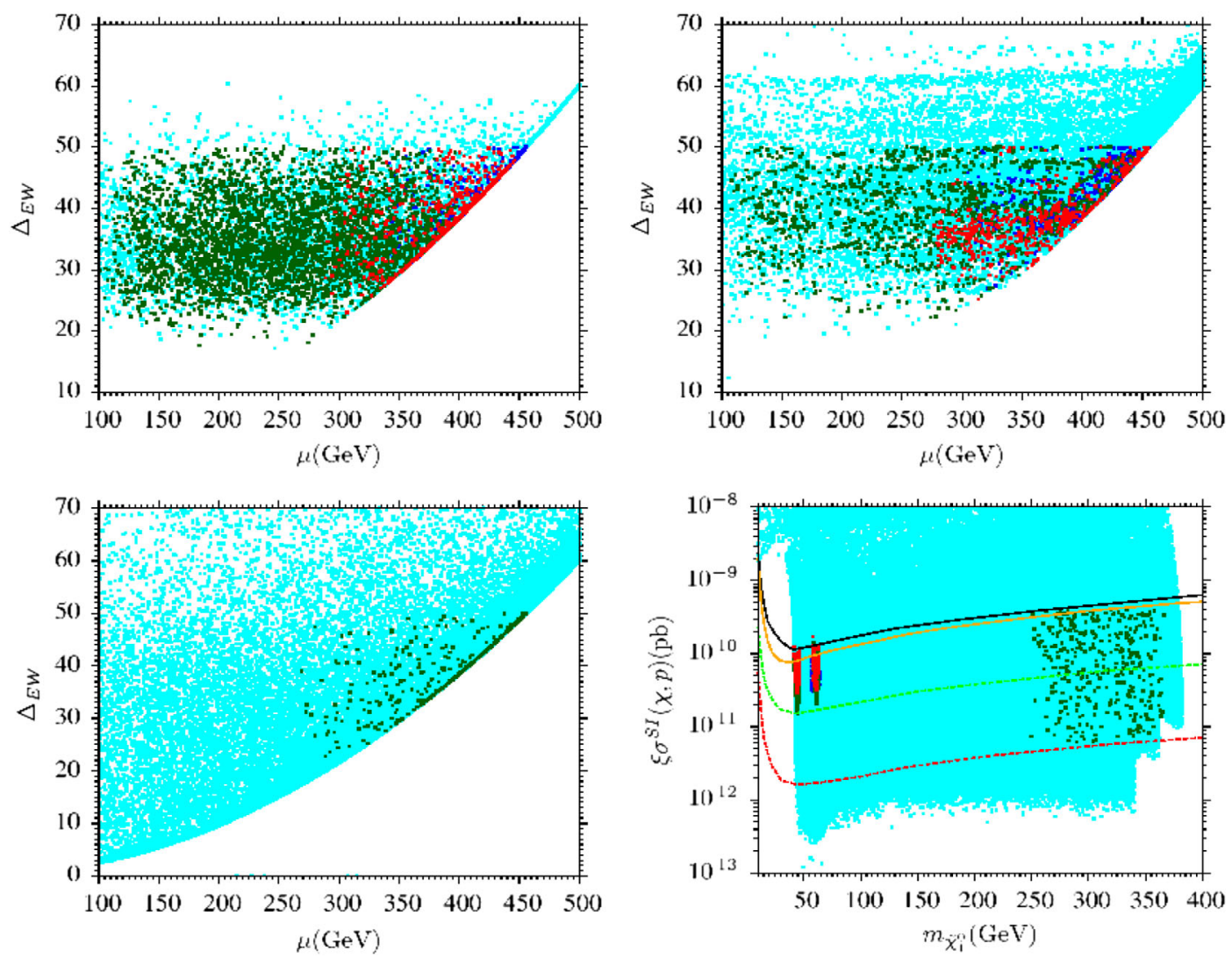

FIG. 1. $\Delta_{\mathrm{EW}}$ vs $\mu$ for the Z-pole (left), for Higgs-pole (right) and Higgsino LSP (bottom left) scenario. Rescaled spin-independent $\left(\xi \sigma^{\mathrm{SI}}(\chi, p)\right)$ rate vs LSP neutralino mass $m_{\tilde{\chi}_{1}^{0}}$ (bottom right). Aqua points satisfy the REWSB and LSP neutralino conditions. Red, blue and green solutions represent the sets of points with relic density consistent with, greater than and smaller than $5 \sigma$ WMAP9 bounds, respectively. These points also satisfy the bounds indicated in Sec. IV.

green points represent the sets of points respectively with DM relic density consistent with, greater than, and smaller than $5 \sigma$ WMAP9 bounds, as well as consistent with upper bounds reported by the LUX experiment. These points all also satisfy the bounds given in Sec. IV. We see that green points both for $Z$-pole and Higgs-pole scenarios have $\Delta_{\mathrm{EW}}$ in the range 20 to 50 , while $\mu$ is in the range of [100, 450] GeV. Blue and red points have $\Delta_{\mathrm{EW}}$ as small as 24 and $\mu$ is confined between $[300,450] \mathrm{GeV}$. In the bottom left panel, we show a plot in the same plane for Higgsino LSP solutions. Because such solutions have small relic densities, all the points are green. One can see that such solutions have $\Delta_{\mathrm{EW}}$ values from 20 to 50 with corresponding $\mu$ values varying roughly from $250 \mathrm{GeV}$ to $450 \mathrm{GeV}$. We will talk about Higgsino LSP solutions more with reference to the plots in $\xi \sigma^{\mathrm{SI}}(\chi, p)-m_{\tilde{\chi}_{1}^{0}}$ plane.

In $\xi \sigma^{\mathrm{SI}}(\chi, p)-m_{\tilde{\chi}_{1}^{0}}$ plot, solid black and red lines respectively represent the current LUX [36] and XENON1T [57] bounds. The dashed green and red lines display projection of XENON1T for next two years and XENONnT (total exposure of 20 t.y) [58], respectively. The factor $\xi \equiv \Omega h^{2} / 0.12$ for green points which accounts for a possible depleted local abundance of neutralino DM, while $\xi=1$ for red and blue points. In this plot, the two dips around $45 \mathrm{GeV}$ and $62 \mathrm{GeV}$ indicate the $Z$-pole and Higgspole solutions. Here, we want to make a comment that in focused scans, we also got points beyond the current LUX bounds but we have chopped them out and have displayed throughout this work only those solutions which are consistent with these bounds. By the way, if we introduce an axino as the LSP, i.e., the lightest neutralino is not the LSP, these chopped points are still natural and consistent with all the current experimental constraints. Moreover, we can see that in the near future the XENON1T experiment will completely probe solutions of our present scans. One can also notice that there is a wide gap between the Higgspole solutions and Higgsino LSP solutions (green points with $m_{\tilde{\chi}_{1}^{0}}$ between 250 and $350 \mathrm{GeV}$ ). We notice that the $\sigma^{\mathrm{SI}}(\chi, p)$ is too high for points with neutralino mass between 65 and $250 \mathrm{GeV}$. Even if we rescale the $\sigma^{\mathrm{SI}}(\chi, p)$, points still rule out by the current LUX bounds. In addition to it, we also notice that for the Higgsino LSP scenario, $\chi_{1}^{0}, \chi_{2}^{0}$, and $\chi_{1}^{ \pm}$are Higgsino dominated, $\chi_{3}^{0}$ is Bino dominated, $\chi_{4}^{0}$ and $\chi_{2}^{ \pm}$are wino dominated. Since $m_{\chi_{1}^{0}} \sim m_{\chi_{2}^{0}} \sim m_{\chi_{1}^{ \pm}}$, leptons from $\chi_{2}^{0}$ and $\chi_{1}^{ \pm}$are hard to reconstruct. The most effective channels that could 
contribute to 3 leptons is $p p \rightarrow \chi_{4}^{0} \chi_{2}^{ \pm}$. However, in this scenario, $m_{\chi_{4}^{0}} \sim \chi_{2}^{ \pm} \leq 520 \mathrm{GeV}$, whereas the ATLAS Collaboration could only exclude points with wino mass smaller than $380 \mathrm{GeV}$ [59]. The CMS Collaboration has better results, but only excludes the points with wino mass smaller than $450 \mathrm{GeV}$ [60]. One can also see [61,62] for probing light Higgsino using monojet searches. This implies that in our case, even though the Higgsino LSP solutions are natural solutions but are out of reach of the present LHC searches. And we have confirmed it from numerical calculations for LHC SUSY searches as well. Therefore, we will not consider them for further analyses.

We want to comment on the light stau-neutralino coannihilation solutions. We find that if we insisting on $\Delta_{\mathrm{EW}} \lesssim 50$, the light stau-neutralino coannihilation scenario is knocked out though it can be achieved if we relax $\Delta_{\mathrm{EW}}$ up to 100 . This is why we will consider only $Z$-pole and Higgs-pole solutions for collider studies.

We have collected five represented benchmark points (BMP) in Table I. BMP1 and BMP2 are the examples

TABLE I. All the masses in this table are in units of GeV. All the points satisfy the constraints described in Sec. IV. BMP1 and BMP2 are examples of $Z$-pole solutions, BMP3 and BMP4 are representatives of Higgs-pole solutions, and BMP 5 is an example for Higgsino LSP solutions.

\begin{tabular}{|c|c|c|c|c|c|}
\hline & BMP 1 & MMP 2 & BMP 3 & BMP 4 & BMP 5 \\
\hline$m_{0}$ & 1449 & 1424 & 1537 & 1367 & 1053 \\
\hline$m_{\tilde{Q}}$ & 1358.3 & 1323.3 & 1404.1 & 1249.4 & 1021.8 \\
\hline$m_{\tilde{U}^{c}}$ & 1765.8 & 1770.3 & 1981.3 & 1760.5 & 1169.3 \\
\hline$m_{\tilde{D}^{c}}$ & 1715.7 & 1701.7 & 1945.9 & 1717.4 & 1189.1 \\
\hline$m_{\tilde{L}}$ & 912.9 & 851.8 & 475.7 & 497.6 & 806.8 \\
\hline$m_{\tilde{E}^{c}}^{L}$ & 756.2 & 607 & 132.2 & 151.3 & 849.1 \\
\hline$M_{1}^{L}$ & 96.81 & 98.19 & 132.6 & 131.1 & 857.7 \\
\hline$M_{2}$ & 812.9 & 751.8 & 1023 & 1105 & 706.8 \\
\hline$M_{3}$ & -977.33 & -882.22 & -1203 & -1329.8 & 1084.1 \\
\hline$A_{t}=A_{b}$ & 3632 & 3689 & 4981 & 5076 & 857.7 \\
\hline$A_{\tilde{\tau}}$ & -403.1 & -413.5 & -238.2 & -186.9 & -2915 \\
\hline $\tan \beta$ & 17.6 & 18.9 & 21.3 & 19.8 & 14.5 \\
\hline$m_{H_{u}}$ & 2631 & 2562 & 3231 & 3306 & 2558 \\
\hline$m_{H_{d}}$ & 2618 & 2697 & 3284 & 3203 & 487.6 \\
\hline$\mu$ & 326 & 254 & 351 & 276 & 262 \\
\hline$\Delta_{\mathrm{EW}}$ & 29 & 24 & 35 & 31 & 29 \\
\hline$\Delta_{\mathrm{HS}}$ & 1691 & 1597 & 2552 & 2660 & 1597 \\
\hline$\Delta a_{\mu}$ & $4.17 \times 10^{-10}$ & $5.59 \times 10^{-10}$ & $6.34 \times 10^{-10}$ & $4.93 \times 10^{-10}$ & $3.61 \times 10^{-10}$ \\
\hline$m_{h}$ & 123 & 123 & 125 & 125 & 123 \\
\hline$m_{H}$ & 2515 & 2553 & 3060 & 3026 & 529 \\
\hline$m_{A}$ & 2499 & 2536 & 3040 & 3006 & 525 \\
\hline$m_{H^{ \pm}}$ & 2516 & 2554 & 3061 & 3027 & 534 \\
\hline$m_{\tilde{\chi}_{1,2}^{0}}$ & $45.9,326$ & 45,255 & 62,355 & 62,283 & 248,271 \\
\hline$m_{\tilde{\chi}_{3,4}^{0}}$ & 337,712 & 266,658 & 363,882 & 287,953 & 373,587 \\
\hline$m_{\tilde{\chi}_{1,2}^{ \pm}}$ & 333,704 & 260,651 & 362,876 & 286, 946 & 265,579 \\
\hline$m_{\tilde{g}}$ & 2220 & 2025 & 2676 & 2918 & 2397 \\
\hline$m_{\tilde{u}_{L, R}}$ & 2374,2542 & 2216,2411 & 2752,2975 & 2873,3026 & 2322,2421 \\
\hline$m_{\tilde{t}_{1,2}}$ & 1173,1731 & 1000,1542 & 1069,1811 & 1046,1960 & 1062,1760 \\
\hline$m_{\tilde{d}_{L, R}}$ & 2375,2561 & 2218,2434 & 2753,3016 & 2875,3047 & 2323,2366 \\
\hline$m_{\tilde{b}_{1,2}}^{u_{L, R}}$ & 1717,2433 & 1525,2287 & 1812,2777 & 1969,2831 & 1734,2285 \\
\hline$m_{\tilde{\nu}_{1,2}}$ & 978 & 878 & 670 & 774 & 1002 \\
\hline$m_{\tilde{\nu}_{3}}$ & 935 & 821 & 532 & 679 & 996 \\
\hline$m_{\tilde{e}_{L, R}}$ & 984, 909 & 883,839 & 683 & 786,522 & 1008,729 \\
\hline$m_{\tilde{\tau}_{1,2}}^{L, R}$ & 816,941 & 719,828 & 264,549 & 162,693 & 716,1001 \\
\hline$\sigma_{\mathrm{SI}}(\mathrm{pb})$ & $8.05 \times 10^{-11}$ & $1.64 \times 10^{-10}$ & $7.33 \times 10^{-11}$ & $1.64 \times 10^{-10}$ & $1.71 \times 10^{-8}$ \\
\hline$\sigma_{\mathrm{SD}}(\mathrm{pb})$ & $1.19 \times 10^{-5}$ & $3.38 \times 10^{-5}$ & $9.07 \times 10^{-6}$ & $2.40 \times 10^{-5}$ & $1.39 \times 10^{-4}$ \\
\hline$\Omega_{\mathrm{CDM}} h^{2}$ & 0.106 & 0.017 & 0.103 & 0.022 & 0.002 \\
\hline
\end{tabular}


of $Z$-pole solutions with correct relic density and small relic density respectively. In these points, gluinos and the first two generations of squarks are heavier than $2 \mathrm{TeV}$, while top and bottom squarks $\tilde{t}_{1,2}$ and $\tilde{b}_{1,2}$ have masses from 1 to $2 \mathrm{TeV}$. The first two generations of sleptons have masses in the range of 800 to $1000 \mathrm{GeV}$ while $\tilde{\tau}_{1,2}$ are in the 720 to $950 \mathrm{GeV}$ mass range. For BMP1 and BMP2, $\Delta_{\mathrm{EW}}$ is 29 and 24 while $m_{\tilde{\chi}_{1}^{ \pm}}$is 333 and $260 \mathrm{GeV}$, respectively. BMP3 and BMP4 represent Higgs-pole solutions with correct relic density and small relic density, respectively. The colored sparticles have masses in same range as for BMP1 and BMP2. The first two generation sleptons are in the mass range 520 to $790 \mathrm{GeV}$ while $\tilde{\tau}_{1,2}$ are in the 160 to $790 \mathrm{GeV}$ range. BMP5 show a Higgsino LSP solution. This point also has similar spectrum as BMP1 with relatively heavy sleptons and winos are heavier than $550 \mathrm{GeV}$. Since these points have very small relic density $\left(\Omega h^{2} \sim 0.002\right)$, we rescale the direct detection rate as $\xi \sigma^{\mathrm{SI}}(\chi, p)$. Moreover, we can see that BMP2, BMP3, and BMP4 have $\Delta a_{\mu}$ within $3 \sigma$ [37].

\section{LHC SEARCHES}

In this section, we examine the constraints from the direct searches for the SUSY particles at the LHC on samples with relic density consistent with or smaller than $5 \sigma$ WMAP9 bounds and also satisfy the current LUX limits on direct detection of LSP neutralino. In order to realize small fine-tuning and satisfy experimental observations simultaneously, only electroweakinos (neutralinos and charginos) and stau are light and could be explored at the current LHC. Therefore, we should consider the following electroweak Drell-Yan production processes:

$$
\begin{aligned}
p p & \rightarrow \tilde{\chi}_{i}^{0} \tilde{\chi}_{j}^{0}, \quad p p \rightarrow \tilde{\chi}_{l}^{ \pm} \tilde{\chi}_{m}^{ \pm}, \\
p p & \rightarrow \tilde{\chi}_{i}^{0} \tilde{\chi}_{l}^{ \pm}, \quad(i, j=2,3,4 \text { and } l, m=1,2) .
\end{aligned}
$$

In general, the produced neutralinos could decay through on-shell or off-shell $Z^{(*)}$ or $h^{(*)}$,

$$
\tilde{\chi}_{i}^{0} \rightarrow Z^{(*)} \tilde{\chi}_{1}^{0}, \quad \tilde{\chi}_{i}^{0} \rightarrow h^{(*)} \tilde{\chi}_{1}^{0},
$$

where the charginos could only decay through $W^{(*)}$,

$$
\tilde{\chi}_{l}^{ \pm} \rightarrow W^{ \pm(*)} \tilde{\chi}_{1}^{0} .
$$

When $\tilde{\tau}_{1}$ is light, such as BMP 3 and BMP 4, new decay modes of $\tilde{\chi}_{i}^{0}$ and $\tilde{\chi}_{l}^{ \pm}$are possible,

$$
\tilde{\chi}_{i}^{0} \rightarrow \tilde{\tau}_{1} \tau, \quad \text { and }, \quad \tilde{\chi}_{l}^{ \pm} \rightarrow \tilde{\tau}_{1} v_{\tau}
$$

and $\tilde{\tau}_{1}$ decay into $\tilde{\chi}_{1}^{0}$ with branching ratio approximates to $100 \%$.

The relevance of different decay modes depends on mass spectra and will significantly influence collider searches for these particles. In Fig. 2, we show branching ratios of $\tilde{\chi}_{2}^{0}$ [Figs. 2(a) and 2(c)] and $\tilde{\chi}_{1}^{ \pm}$[Figs. 2(b) and 2(d))] for samples considered in this work. The dominant decay channel of $\tilde{\chi}_{2}^{0}$ for samples of $Z$ pole is $\tilde{\chi}_{2}^{0} \rightarrow \tilde{\chi}_{1}^{0} Z^{(*)}$ when the mass difference $m_{\tilde{\chi}_{2}^{0}}-m_{\tilde{\chi}_{1}^{0}}$ is small. Once the decay into Higgs boson is kinematically possible, branching ratio to $\tilde{\chi}_{1}^{0} h$ increase with increasing of $m_{\tilde{\chi}_{2}^{0}}-m_{\tilde{\chi}_{1}^{0}}$ and become the dominant channel when $m_{\tilde{\chi}_{2}^{0}}-m_{\tilde{\chi}_{1}^{0}} \gtrsim 140 \mathrm{GeV}$. The decay channels of $\tilde{\chi}_{1}^{ \pm}$is always $\tilde{\chi}_{1}^{ \pm} \rightarrow \tilde{\chi}_{1}^{0} W^{(*)}$. For samples of the Higgs pole, situations are more complex due to light $\tilde{\tau}_{1}$, as can be seen from BMP 3 and BMP4. The decay of $\tilde{\chi}_{2}^{0}$ to $\tilde{\tau}_{1} \tau$ would be significant or even dominant 2(c).

ATLAS $[63,64]$ and CMS [60] have performed electroweakinos searches for wino $\tilde{\chi}_{2}^{0} / \tilde{\chi}_{1}^{ \pm}$with particular decay models. We use the powerful package CheckMate [65-67] (where PYTHIA 8 [68] and a tuned version of DELPHES 3 [69] have been used internally) to implement LHC constraints. ${ }^{1}$ NLO production rates are obtained by rescaling LO rates with K-factors calculated by Prospino 2 [70], which yield about 1.2 for Higgsino pair production.

As for electroweakinos searches, currently CheckMate has only employed the ATLAS analyses with $13.3 \mathrm{fb}^{-1}$ data [63]. So in order to fully take into account the current constraints, we also recast the latest ATLAS [64] and CMS [60] analyses based on a Monte Carlo simulation. In the simulation, MadGraph 5 [71] is adopted to generate background and signal samples, and PYTHIA 6 [72] is employed to handle the parton shower, particle decay, and hadronization processes. We use MLM scheme to deal with the matching between matrix element and parton shower calculations, and use Delphes 3 [69] to carry out a fast detection simulation with the CMS setup. Jets are reconstructed using the anti- $k_{T}$ algorithm [73] with a distance parameter $\Delta R=0.4$.

Generally, heavy electroweakino productions with successive decay will lead to multilepton signals; among them, $3 l+E_{\mathrm{T}}^{t}$ and $2 l+E_{\mathrm{T}}^{\prime}$ give the best sensitivity at the LHC searches. In the case of the $3 l+E_{\mathrm{T}}$ search channel, major SM backgrounds are $Z Z$ and $W Z$ productions. Two leptons from $Z$ decay are required to form sameflavor-opposite-sign (SFOS) pair. Two useful kinematic variables to discriminate signals from backgrounds are $m_{\mathrm{T}}$ and $E_{\mathrm{T}}^{\dagger}$, where $m_{\mathrm{T}}$ is the transverse mass defined as $m_{\mathrm{T}}=\sqrt{2\left(p_{\mathrm{T}}^{l} E_{\mathrm{T}}-\mathbf{p}_{\mathrm{T}}^{l} \cdot \mathbf{p}_{\mathrm{T}}^{\text {miss }}\right)}$ with $p_{\mathrm{T}}^{\text {miss }}$ the missing transverse momentum vector, and the lepton $l$ as the one not forming the SFOS lepton pair.

In Fig. 3, we present the $m_{\mathrm{T}}$ and $E_{\mathrm{T}}$ distributions of backgrounds and signals. In the case of $Z Z$ background, each $Z$ decays to $l^{+} l^{-}$pair but one of the leptons out of four lepton can not be reconstructed successfully. In this way one

\footnotetext{
${ }^{1}$ Some results of ATLAS and/or CMS involving tau final states cannot be well reproduced by the CheckMATE, in this case we use our own codes to set constraints. Details about analysis and corresponding validation processes are given in Appendix.
} 


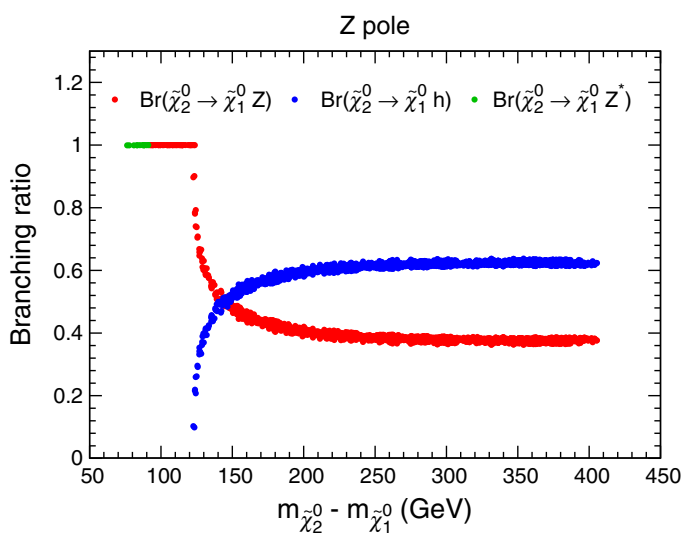

(a) $\tilde{\chi}_{2}^{0}$ decay branching ratio, $\mathrm{Z}$ pole.

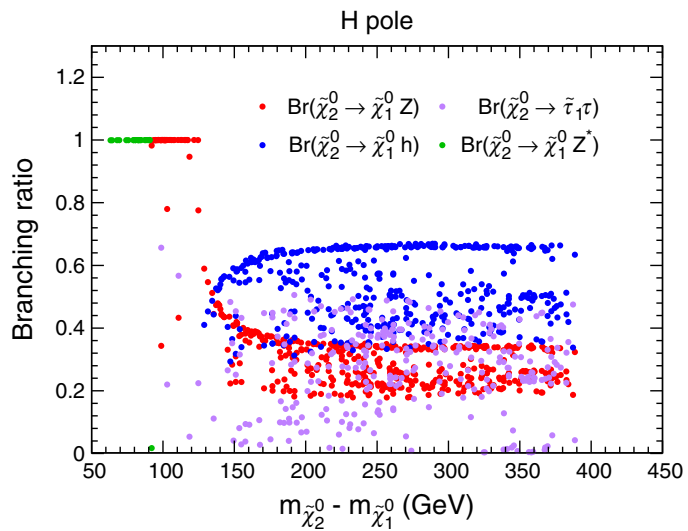

(c) $\tilde{\chi}_{2}^{0}$ decay branching ratio, $\mathrm{H}$ pole.

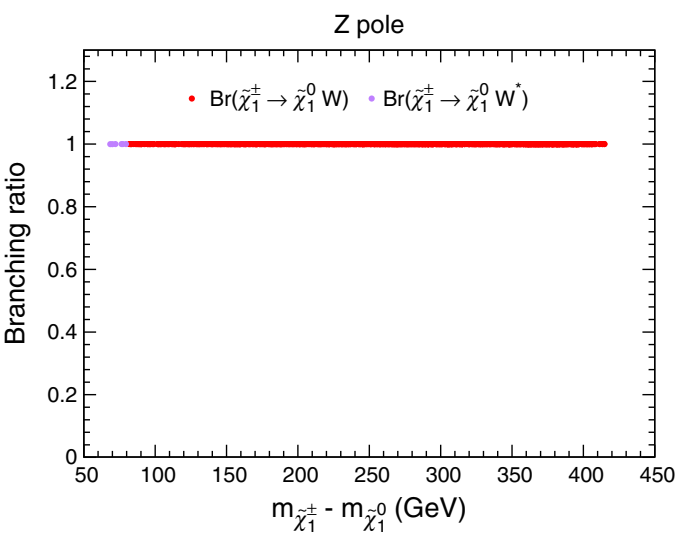

(b) $\tilde{\chi}_{1}^{ \pm}$decay branching ratio, $\mathrm{Z}$ pole.

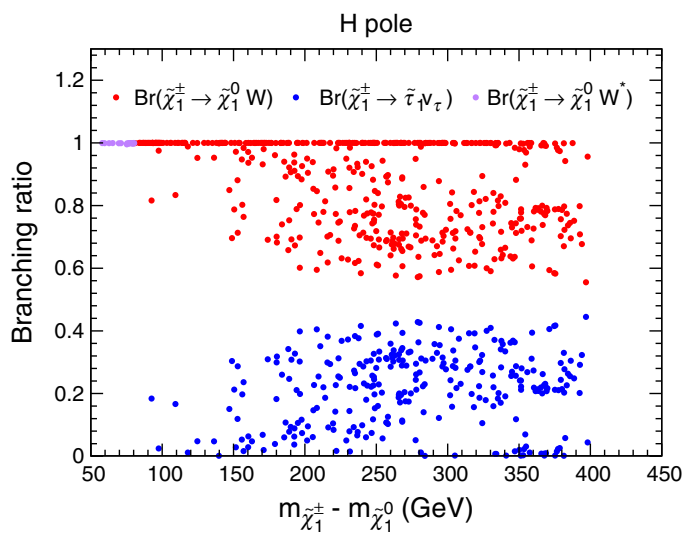

(d) $\tilde{\chi}_{1}^{ \pm}$decay branching ratio, $\mathrm{H}$ pole.

FIG. 2. Decay branching ratios of $\tilde{\chi}_{2}^{0}$ (a and b) and $\tilde{\chi}_{1}^{ \pm}$(c and d) for samples with relic density consistent with or smaller than $5 \sigma$ WMAP9 bounds.

gets the $3 l$ final state. As there is no neutrino contributing $E_{\mathrm{T}}$, its $E_{\mathrm{T}}$ distribution is softer than others, and so is its $m_{\mathrm{T}}$ distribution. For the $W Z$ background, the $m_{\mathrm{T}}$ variable is bounded by the $W$ boson mass, leading to an obvious endpoint near $m_{W}$. All $m_{\mathrm{T}}$ and $E_{\mathrm{T}}$ for signals are harder and are easy to be distinguished from backgrounds.

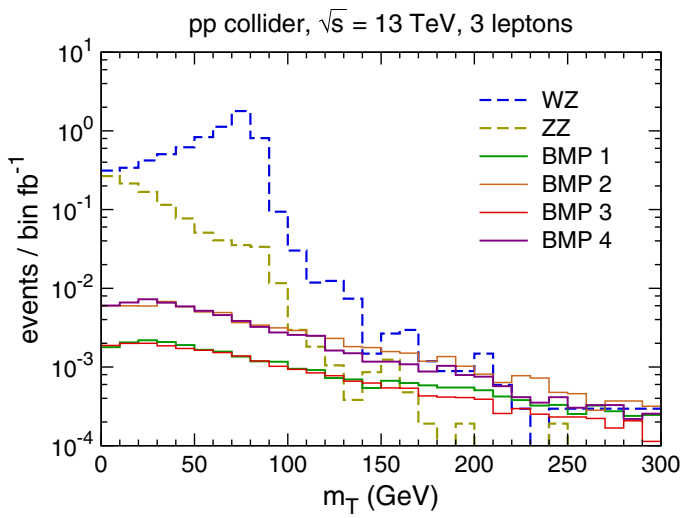

(a) $m_{\mathrm{T}}$ distributions
In the case of $2 l+E_{\mathrm{T}}$ search channel, dominant backgrounds are $W Z, W W, Z Z$, and $t \bar{t}$ production. Still, these two leptons from $Z$ decay are required to form SFOS pairs, whose invariable is a useful variable to distinguish signals from SM backgrounds. Another useful variable $m_{\mathrm{T} 2}$ is defined as

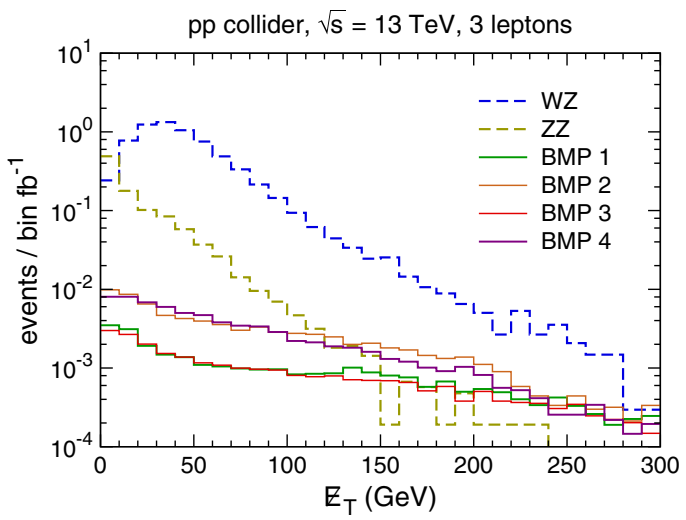

(b) $\mathbb{E}_{\mathrm{T}}$ distributions

FIG. 3. $m_{\mathrm{T}}\left(\right.$ a) and $\mathscr{E}_{\mathrm{T}}$ (b) distributions for backgrounds and signal benchmark points in the $3 l+E_{\mathrm{T}}$ channel at the $13 \mathrm{TeV} \mathrm{LHC}$. 


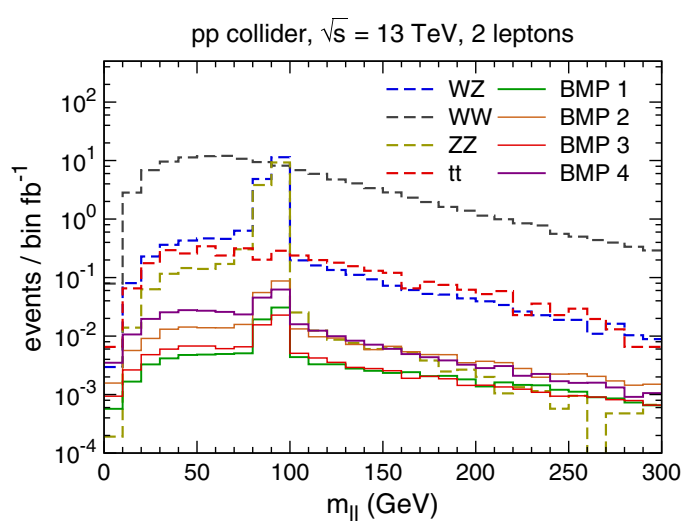

(a) $\mathrm{m}_{l l}$ distributions

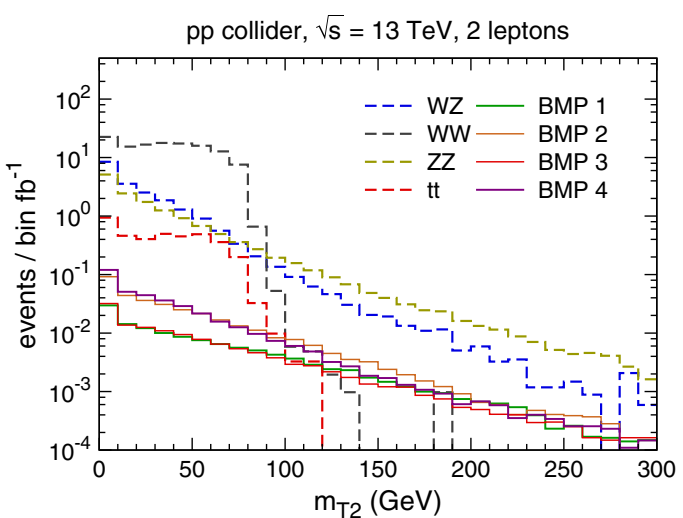

(b) $m_{\mathrm{T} 2}$ distributions

FIG. 4. $\quad \mathrm{m}_{l l}$ (a) and $m_{\mathrm{T} 2}$ (b) distributions for backgrounds and signal benchmark points in the $2 l+E_{\mathrm{T}}$ channel at the $13 \mathrm{TeV} \mathrm{LHC}$.

$$
m_{\mathrm{T} 2}=\min _{\mathbf{p}_{\mathrm{T}}^{1}+\mathbf{p}_{\mathrm{T}}^{2}=\mathbf{p}_{\mathrm{T}}^{\mathrm{miss}}}\left\{\max \left[m_{\mathrm{T}}\left(\mathbf{p}_{\mathrm{T}}^{a}, \mathbf{p}_{\mathrm{T}}^{1}\right), m_{\mathrm{T}}\left(\mathbf{p}_{\mathrm{T}}^{b}, \mathbf{p}_{\mathrm{T}}^{2}\right)\right]\right\},
$$

where $m_{\mathrm{T}}\left(\mathbf{p}_{\mathrm{T}}^{i}, \mathbf{p}_{\mathrm{T}}^{j}\right)=\sqrt{2\left(p_{\mathrm{T}}^{i} p_{\mathrm{T}}^{j}-\mathbf{p}_{\mathrm{T}}^{i} \cdot \mathbf{p}_{\mathrm{T}}^{j}\right)}$, and $\mathbf{p}_{\mathrm{T}}^{a}$ and $\mathbf{p}_{\mathrm{T}}^{b}$ are the transverse momenta of two visible particles in the decay chain (two leptons in our case). $\mathbf{p}_{\mathrm{T}}^{1}$ and $\mathbf{p}_{\mathrm{T}}^{2}$ are a partition of the missing transverse momentum $\mathbf{p}_{\mathrm{T}}^{\text {miss }}$. By definition, $m_{\mathrm{T} 2}$ is the minimum of the larger $m_{\mathrm{T}}$ over all partitions, its distribution for two identical chains has an upper endpoint, which is determined by the mass difference between the parent particle and its invisible child.

In Fig. 4, we demonstrate the $\mathrm{m}_{l l}$ and $m_{\mathrm{T} 2}$ distributions of backgrounds and signals. For the $W Z$ and $Z Z$ backgrounds and signals, lepton pairs from $Z$ boson decay result in peaks around $m_{Z}$ in the $\mathrm{m}_{l l}$ distributions, as shown in Fig. 4(a). Whereas these two leptons for $W W$ and $t \bar{t}$ backgrounds origin from two particles and do not have obvious feature. Fortunately, the $m_{\mathrm{T} 2}$ distributions for the $W W$ and $t \bar{t}$ backgrounds are essentially bounds by $m_{W}$.

For the analyses of Ref. [63], we use CheckMATE to calculate the corresponding significance. And for the analyses of Refs. [60] and Ref. [64], we apply the same cuts in

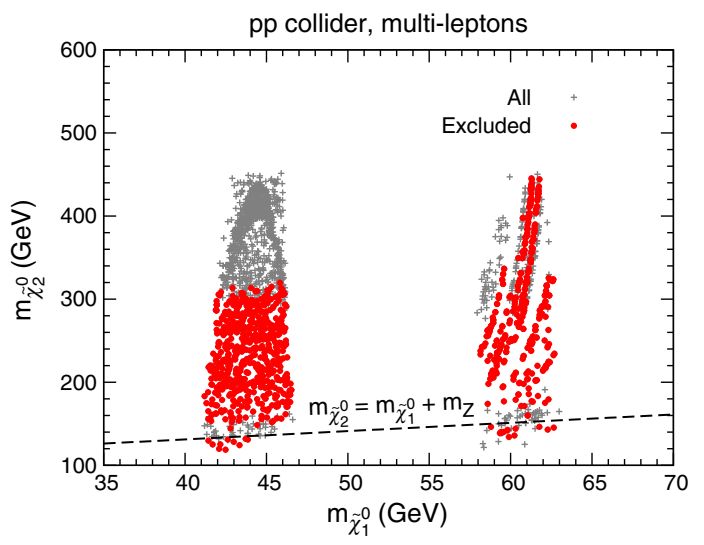

(a) $m_{\tilde{\chi}_{1}^{0}-} m_{\tilde{\chi}_{2}^{0}}$ plane various signal regions, and compare the obtained cross sections to $95 \%$ limits tabulated in these literatures. To justify our simulations and recast procedures, we have reproduced the exclusion limits in experimental papers and found good agreement. In Fig. 5, we present the 95\% C.L. exclusion results of the LHC electroweakino searches in the $m_{\tilde{\chi}_{1}^{0}}-m_{\tilde{\chi}_{2}^{0}}$ plane [Fig. 5(a)] and $m_{\tilde{\chi}_{1}^{0}}-\Delta_{\text {EW }}$ plane [Fig. 5(b)]. $3 l+E_{\mathrm{T}}$ searches require exactly three hard leptons. As a result, samples close to $Z$ threshold, i.e., $m_{\tilde{\chi}_{2}^{0}} \sim m_{\tilde{\chi}_{1}^{0}}+m_{Z}$, are hard to explore. Points below this threshold would be excluded by $2 l+E_{\mathrm{T}}$ searches due to small $m_{\tilde{\chi}_{1}^{ \pm}}$and large production cross sections.

Roughly, $3 l+E_{\mathrm{T}}^{\prime}$ and $2 l+E_{\mathrm{T}}^{6}$ searches at the current LHC could exclude Higgsino dominant $\tilde{\chi}_{2}^{0} / \tilde{\chi}_{1}^{ \pm}$with mass up to $300 \mathrm{GeV}$. This result is consistent with our previous prediction [74] whereas seems to somewhat weaker than that given by the ATLAS [63] and CMS Collaborations [60]. The main reason is that in their searches, pure wino $\tilde{\chi}_{2}^{0} / \tilde{\chi}_{1}^{ \pm}$is assumed, which have larger production cross sections. Besides, they assume $\tilde{\chi}_{1}^{ \pm}$and $\tilde{\chi}_{2}^{0}$ decay via $W^{*}$ and $Z^{*}$ bosons with a branching fraction of $100 \%$, whereas decay branching ratios in our samples highly depend on mass spectra.

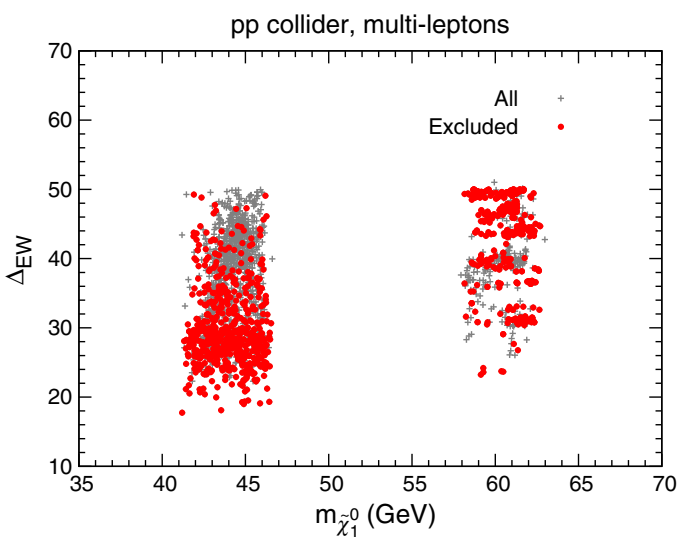

(b) $m_{\tilde{\chi}_{1}^{0}}-\Delta_{\text {EW }}$ plane

FIG. 5. $95 \%$ C.L. exclusion results of the LHC electroweakinos searches in the $m_{\tilde{\chi}_{1}^{0}}-m_{\tilde{\chi}_{2}^{0}}$ plane (a) and $m_{\tilde{\chi}_{1}^{0}}-\Delta_{\text {EW }}$ plane (b). 
Another and even stricter constraint on samples of Higgs pole come from the searches for electroweakinos with tau final states. These searches have been performed by both CMS [60] and ATLAS [64] Collaborations, by latter it was shown that when $\tilde{\chi}_{2}^{0} / \tilde{\chi}_{1}^{ \pm}$decay into $\tilde{\chi}_{1}^{0}$ via an intermediate on-shell stau or tau sneutrino, $\tilde{\chi}_{2}^{0} / \tilde{\chi}_{1}^{ \pm}$with mass up to $760 \mathrm{GeV}$ are excluded for a massless $\tilde{\chi}_{1}^{0}$. In our case samples with $\tilde{\chi}_{2}^{0}$ mass up to $460 \mathrm{GeV}$ for Higgs pole could still be excluded, as shown in Fig. 5(a).

Finally, we project exclusion results into $m_{\tilde{\chi}_{1}^{0}}-\Delta_{\mathrm{EW}}$ plane [Fig. 5(b)]. Samples of $Z$ pole with small $\Delta_{\mathrm{EW}}$ are easy to be explored, whereas these with large $\Delta_{\mathrm{EW}}$ are hard to be excluded due to large $\mu$, which in turn indicate large $m_{\tilde{\chi}_{2}^{0}}$ and small production cross sections. In the case of Higgs pole, many samples with $\Delta_{\mathrm{EW}}$ up to 50 could by excluded by electroweakinos searches with tau final states. For both the $Z$ pole and Higgs pole, samples with $\Delta_{\mathrm{EW}}$ approximate to 20 could still survive, indicating naturalness of this SUSY framework.

\section{DISCUSSIONS AND CONCLUSION}

We have studied natural supersymmetry in the GmSUGRA, and found that after demanding $\Delta_{\text {EW }} \lesssim 50$, only the parameter space related to $Z$-pole and Higgs-pole solutions are left. We performed the focused scans for such parameter space and showed that it satisfies various phenomenological constraints and is compatible with the current direct detection bound on neutralino DM reported by the LUX experiment. Such parameter space also has solutions with the correct DM relic density besides the solutions with relic density smaller or larger than $5 \sigma$ WMAP9 bounds. We also performed the collider study of such solutions by implementing and comparing with relevant studies done by the ATLAS and CMS Collaborations. We showed that the points with the Higgsino dominant $\tilde{\chi}_{2}^{0} / \tilde{\chi}_{1}^{ \pm}$mass up to $300 \mathrm{GeV}$ are

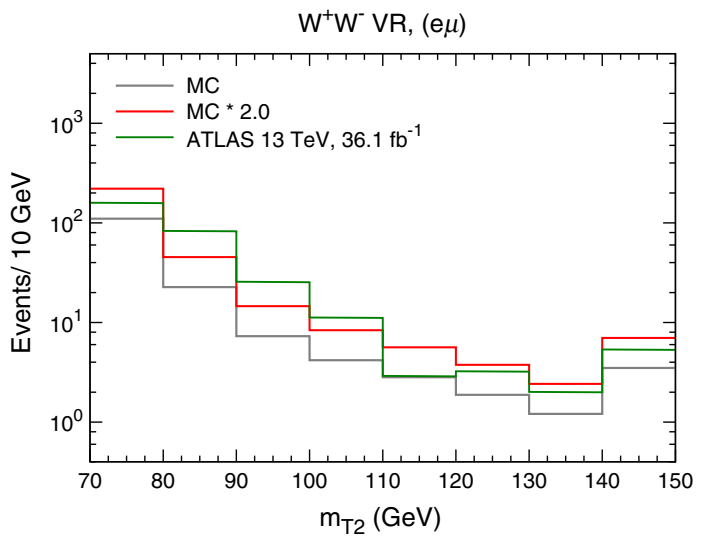

(a) $W W$ validation region excluded for $Z$ pole scenario while for Higgs-pole scenario, points with $\tilde{\chi}_{2}^{0}$ mass up to $460 \mathrm{GeV}$ are excluded. Next, we displayed that both for the Z-pole and Higgs-pole scenarios, the points having $\Delta_{\mathrm{EW}} \sim 20$ still survive. Moreover, we present five benchmark points as examples of our present scans. In these benchmark points, gluino and the first two generations of squarks are heavier than $2 \mathrm{TeV}$, top squarks $\tilde{t}_{1,2}$ are in the mass range $[1,2] \mathrm{TeV}$, while sleptons are lighter than $1 \mathrm{TeV}$. We also discuss that stau-neutralino coannihilation scenario is not compatible with our demand of $\Delta_{\mathrm{EW}} \lesssim 50$. On the other hand, Higgsino LSP solutions are natural solutions but out of reach of present LHC searches. Some part of the parameter space can explain the anomaly of muon $(g-2)_{\mu}$ within $3 \sigma$ as well.

\section{ACKNOWLEDGMENTS}

X. J. B. and P. F. were supported by the National Natural Science Foundation of China under Grants No. 11475189 and No. 11475191 and the National Key Program for Research and Development (No. 2016YFA0400200). T. L. was supported in part by Projects No. 11475238 and No. 11647601 of the National Natural Science Foundation of China and by the Key Research Program of Frontier Science, CAS, and the CAS-TWAS Presidents Fellowship Programme (W. A.). The numerical results described in this paper have been obtained via the HPC Cluster of ITP-CAS. S. R. thanks T. L. for warm hospitality and the Institute of Theoretical Physics, CAS, Beijing, China, for providing a conducive atmosphere for research while part of this work was completed.

\section{APPENDIX: VALIDATION OF ANALYSES INVOLVING TAU FINAL STATES}

In this appendix, we provide and validate analyses involving tau final states [64]. We use MadGraph 5 to

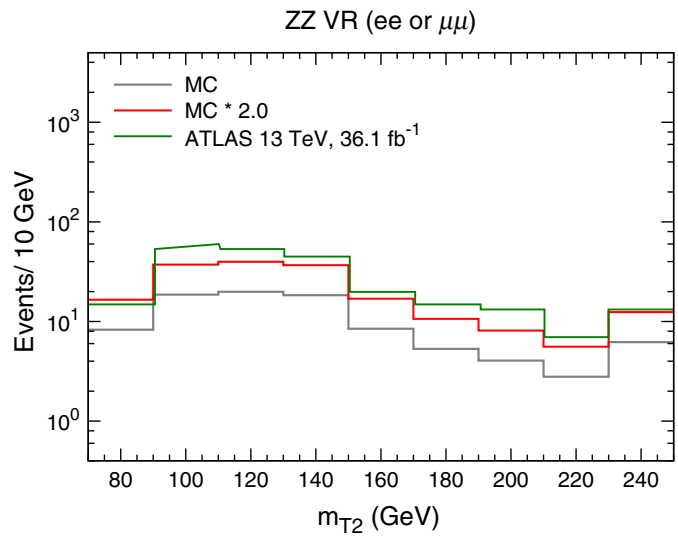

(b) $Z Z$ validation region

FIG. 6. The $m_{\mathrm{T} 2}$ distributions in the WW-VR (Left) and ZZ-VR (right) regions. Green lines are distributions given by ATLAS, while gray lines are our distributions based on MC tools. After multiplying a factor of 2, our results are consistent with ATLAS. 
generate standard model (SM) diboson background ( $Z Z$, $W Z$, and $W W$ ), and impose the cuts of $W W$ validation region and $Z Z$ validation region (Table 7 in Ref. [64]). Gray lines in Fig. 6 are $m_{\mathrm{T} 2}$ distributions in these two validation regions. Green lines are distributions presented in Ref. [64]. Obviously, there is a discrepancy between our $m_{\mathrm{T} 2}$ and those of ATLAS. However, their shapes are very similar. After multiplying a factor of 2, our results are almost consistent with ATLAS. This factor and the discrepancy after timing this factor can be contributed to many reasons. First, we use different MC generators and we do not include higher-order corrections to SM processes. Besides, we use Delphes 3 to do the detector simulation, while ATLAS adopt GEANT 4. We take this factor as our MC bias and use it to normalize backgrounds and signals.

Signal processes $p p \rightarrow \tilde{\chi}_{1}^{ \pm} \tilde{\chi}_{1}^{0}$ are also generated by using MadGraph 5. After detector simulation, we impose the cuts of signal regions (Table 1 of Ref. [64]) and obtain corresponding visible cross sections. Points are excluded when their visible cross sections are larger than $\sigma_{\text {vis }}^{95}$. When set limits on signal samples, we multiply the signal production cross sections by the bias factor along with K-factor. Fig. 7 is the exclusion results in the $\tilde{\chi}_{1}^{ \pm}-\tilde{\chi}_{1}^{0}$ plane.

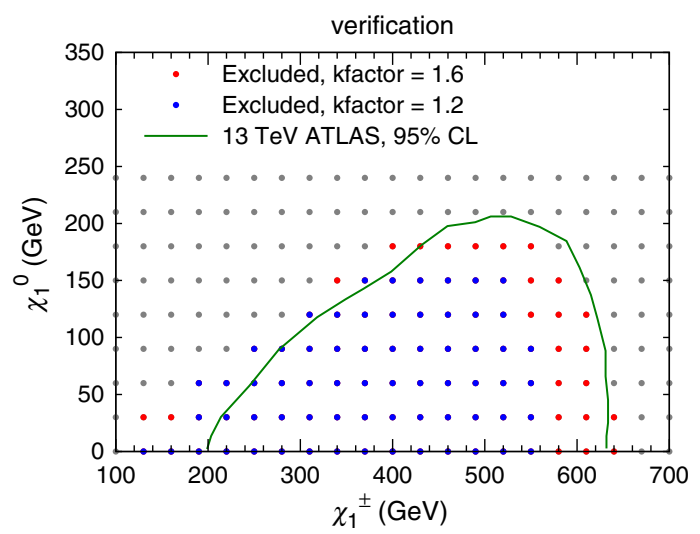

FIG. 7. The $95 \% \mathrm{CL}$ exclusion results for $\tilde{\chi}^{+} \tilde{\chi}^{-}$production. Green line is the limit of ATLAS [64]. Red and blue points are excluded by our analyses with K-factor equal to 1.6 and 1.2 respectively.

It is obvious that when the K-factor equals to 1.6 , we can repeat the results of ATLAS. In the realistic situation, we use Prospino 2 [70] to calculate the K-factor, which yields about 1.2 for Higgsino pair production.
[1] S. Dimopoulos, S. Raby, and F. Wilczek, Phys. Rev. D 24, 1681 (1981); U. Amaldi, W. de Boer, and H. Furstenau, Phys. Lett. B 260, 447 (1991); J. R. Ellis, S. Kelley, and D. V. Nanopoulos, Phys. Lett. B 260, 131 (1991); P. Langacker and M. X. Luo, Phys. Rev. D 44, 817 (1991).

[2] E. Witten, Nucl. Phys. B188, 513 (1981); R. K. Kaul, Phys. Lett. B 109, 19 (1982).

[3] H. E. Haber and R. Hempfling, Phys. Rev. Lett. 66, 1815 (1991); J. R. Ellis, G. Ridolfi, and F. Zwirner, Phys. Lett. B 257, 83 (1991); Y. Okada, M. Yamaguchi, and T. Yanagida, Prog. Theor. Phys. 85, 1 (1991); For a review, see e.g., M. S. Carena and H. E. Haber, Prog. Part. Nucl. Phys. 50, 63 (2003).

[4] G. Aad et al. (ATLAS Collaboration), Phys. Lett. B 716, 1 (2012).

[5] S. Chatrchyan et al. (CMS Collaboration), Phys. Lett. B 716, 30 (2012).

[6] H. Goldberg, Phys. Rev. Lett. 50, 1419 (1983); J. Ellis, J. Hagelin, D. V. Nanopoulos, K. Olive, and M. Srednicki, Nucl. Phys. B238, 453 (1984).

[7] For reviews, see G. Jungman, M. Kamionkowski, and K. Griest Phys. Rep., 267, 195 (1996); K. A. Olive, arXiv: astro-ph/0301505; J. L. Feng, Ann. Phys. 315, 2 (2005); M. Drees, AIP Conf. Proc. 805, 48 (2005); J. L. Feng, Annu. Rev. Astron. Astrophys. 48, 495 (2010).

[8] ATLAS Collaboration, Report No. ATLAS-CONF-2017022; CMS Collaboration, Report No. CMS-SUS-16-036.

[9] M. Drees and J. S. Kim, Phys. Rev. D 93, 095005 (2016).
[10] R. Ding, T. Li, F. Staub, and B. Zhu, Phys. Rev. D 93, 095028 (2016).

[11] H. Baer, V. Barger, and M. Savoy, Phys. Rev. D 93, 035016 (2016).

[12] B. Batell, G. F. Giudice, and M. McCullough, J. High Energy Phys. 12 (2015) 1.

[13] S. S. AbdusSalam and L. Velasco-Sevilla, Phys. Rev. D 94, 035026 (2016).

[14] D. Barducci, A. Belyaev, A. K. M. Bharucha, W. Porod, and V. Sanz, J. High Energy Phys. 07 (2015) 066.

[15] T. Cohen, J. Kearney, and M. Luty, Phys. Rev. D 91, 075004 (2015).

[16] J. Fan, M. Reece, and L. T. Wang, J. High Energy Phys. 08 (2015) 152.

[17] T. Leggett, T. Li, J. A. Maxin, D. V. Nanopoulos, and J. W. Walker, arXiv:1403.3099; Phys. Lett. B 740, 66 (2015).

[18] S. Dimopoulos, K. Howe, and J. March-Russell, Phys. Rev. Lett. 113, 111802 (2014).

[19] I. Gogoladze, F. Nasir, and Q. Shafi, J. High Energy Phys. 11 (2013) 173.

[20] G. D. Kribs, A. Martin, and A. Menon, Phys. Rev. D 88, 035025 (2013).

[21] I. Gogoladze, F. Nasir, and Q. Shafi, Int. J. Mod. Phys. A 28, 1350046 (2013).

[22] G. Du, T. Li, D. V. Nanopoulos, and S. Raza, Phys. Rev. D 92, 025038 (2015).

[23] E. Cremmer, S. Ferrara, C. Kounnas, and D. V. Nanopoulos, Phys. Lett. B 133, 61 (1983); J. R. Ellis, A. B. Lahanas, 
D. V. Nanopoulos, and K. Tamvakis, Phys. Lett. B 134, 429 (1984); J. R. Ellis, C. Kounnas, and D. V. Nanopoulos, Nucl. Phys. B241, 406 (1984); B247, 373 (1984); A. B. Lahanas and D. V. Nanopoulos, Phys. Rep. 145, 1 (1987).

[24] G. F. Giudice and A. Masiero, Phys. Lett. B 206, 480 (1988).

[25] T. Li, S. Raza, and X. C. Wang, Phys. Rev. D 93, 115014 (2016).

[26] H. Baer, V. Barger, J. S. Gainer, H. Serce, and X. Tata, Phys. Rev. D 96, 115008 (2017); P. Fundira and A. Purves, Int. J. Mod. Phys. A 33, 1841004 (2018); J. Cao, X. Guo, Y. He, L. Shang, and Y. Yue, Phys. Rev. D 96, 035038 (2017); B. Zhu, F. Staub, and R. Ding, Phys. Rev. D 96, 035038 (2017); M. Abdughani, L. Wu, and J. M. Yang, Eur. Phys. J. C 78, 4 (2018); L. Wu, Eur. Phys. J. C 77, 868 (2017); H. Baer, V. Barger, M. Savoy, H. Serce, and X. Tata, J. High Energy Phys. 06 (2017) 101; K. J. Bae, H. Baer, and H. Serce, J. Cosmol. Astropart. Phys. 06 (2017) 024; T. r. Liang, B. Zhu, R. Ding, and T. Li, Adv. High Energy Phys. 2017, 1585023 (2017); C. Li, B. Zhu, and T. Li, Nucl. Phys. B 927, 255 (2018); P.S. B. Dev, C. M. Vila, and W. Rodejohann, arXiv:1703.00828; H. Baer, V. Barger, J.S. Gainer, P. Huang, M. Savoy, H. Serce, and X. Tata, Phys. Lett. B 774, 451 (2017); ; L. Delle Rose, S. Khalil, S. J. D. King, C. Marzo, S. Moretti, and C. S. Un, Phys. Rev. D 96, 055004 (2017); G. G. Ross, K. Schmidt-Hoberg, and F. Staub, J. High Energy Phys. 03 (2017) 021; L. Calibbi, T. Li, A. Mustafayev, and S. Raza, Phys. Rev. D 93, 115018 (2016); M. van Beekveld, W. Beenakker, S. Caron, R. Peeters, and R. Ruiz de Austri, Phys. Rev. D 96, 035015 (2017); M. Peiro and S. Robles, J. Cosmol. Astropart. Phys. 05 (2017) 010; A. Kaminska, G. G. Ross, and K. SchmidtHoberg, J. High Energy Phys. 11 (2013) 209; D. Horton and G. G. Ross, Nucl. Phys. B830, 221 (2010).

[27] J. R. Ellis, K. Enqvist, D. V. Nanopoulos, and F. Zwirner, Mod. Phys. Lett. A 01, 57 (1986);

[28] R. Barbieri and G. F. Giudice, Nucl. Phys. B306, 63 (1988).

[29] H. Baer, V. Barger, P. Huang, A. Mustafayev, and X. Tata, Phys. Rev. Lett. 109, 161802 (2012).

[30] H. Baer, V. Barger, P. Huang, D. Mickelson, A. Mustafayev, and X. Tata, Phys. Rev. D 87, 035017 (2013).

[31] E. Hardy, J. High Energy Phys. 10 (2013) 133.

[32] W. Ahmed, L. Calibbi, T. Li, A. Mustafayev, and S. Raza, Phys. Rev. D 95, 095031 (2017).

[33] T. Li and D. V. Nanopoulos, Phys. Lett. B 692, 121 (2010).

[34] T. Li and S. Raza, Phys. Rev. D 91, 055016 (2015).

[35] T. Li, S. Raza, and K. Wang, Phys. Rev. D 93, 055040 (2016).

[36] D. S. Akerib et al. (LUX Collaboration), Phys. Rev. Lett. 118, 021303 (2017).

[37] G. W. Bennett et al. (Muon G-2 Collaboration), Phys. Rev. D 73, 072003 (2006); G. W. Bennett et al. (Muon (g-2) Collaboration), Phys. Rev. D 80, 052008 (2009).

[38] T. Cheng, J. Li, T. Li, D. V. Nanopoulos, and C. Tong, Eur. Phys. J. C 73, 2322 (2013).

[39] C. Balazs, T. Li, D. V. Nanopoulos, and F. Wang, J. High Energy Phys. 09 (2010) 003.

[40] H. Baer, F. E. Paige, S. D. Protopopescu, and X. Tata, arXiv: hep-ph/0001086.
[41] J. Hisano, H. Murayama, and T. Yanagida, Nucl. Phys. B402, 46 (1993); Y. Yamada, Z. Phys. C 60 (1993) 83; J. L. Chkareuli and I. G. Gogoladze, Phys. Rev. D 58, 055011 (1998).

[42] D. M. Pierce, J. A. Bagger, K. T. Matchev, and R.-j. Zhang, Nucl. Phys. B491, 3 (1997).

[43] L. E. Ibanez and G. G. Ross, Phys. Lett. B 110, 215 (1982); K. Inoue, A. Kakuto, H. Komatsu, and S. Takeshita, Prog. Theor. Phys. 68, 927 (1982); 70, 330(E) (1983); L. E. Ibanez, Phys. Lett. B 118, 73 (1982); J. R. Ellis, D. V. Nanopoulos, and K. Tamvakis, Phys. Lett. B 121, 123 (1983); L. Alvarez-Gaume, J. Polchinski, and M. B. Wise, Nucl. Phys. B221, 495 (1983).

[44] J. Beringer et al. (Particle Data Group Collaboration), Phys. Rev. D 86, 010001 (2012).

[45] Tevatron Electroweak Working Group and CDF Collaboration and D0 Collaboration, arXiv:0903.2503.

[46] I. Gogoladze, R. Khalid, S. Raza, and Q. Shafi, J. High Energy Phys. 06 (2011) 117.

[47] G. Belanger, F. Boudjema, A. Pukhov, and R. K. Singh, J. High Energy Phys. 11 (2009) 026; H. Baer, S. Kraml, S. Sekmen, and H. Summy, J. High Energy Phys. 03 (2008) 056.

[48] G. Aad et al. (ATLAS and CMS Collaborations), J. High Energy Phys. 08 (2016) 045.

[49] B. C. Allanach, A. Djouadi, J. L. Kneur, W. Porod, and P. Slavich, J. High Energy Phys. 09 (2004) 044.

[50] K. J. Bae, H. Baer, N. Nagata, and H. Serce, Phys. Rev. D 92, 035006 (2015).

[51] A. Pierce, N. R. Shah, and S. Vogl, Phys. Rev. D 97, 023008 (2018).

[52] H. Baer and M. Brhlik, C. h. Chen, and X. Tata, Phys. Rev. D 55, 4463 (1997); H. Baer, M. Brhlik, D. Castano, and X. Tata, Phys. Rev. D 58, 015007 (1998).

[53] K. S. Babu and C. F. Kolda, Phys. Rev. Lett. 84, 228 (2000); A. Dedes, H. K. Dreiner, and U. Nierste, Phys. Rev. Lett. 87, 251804 (2001); J. K. Mizukoshi, X. Tata, and Y. Wang, Phys. Rev. D 66, 115003 (2002).

[54] V. Khachatryan et al. (CMS and LHCb Collaborations), Nature (London) 522, 68 (2015).

[55] Y. Amhis et al. (Heavy Flavor Averaging Group (HFAG) Collaboration), arXiv:1412.7515.

[56] C. L. Bennett et al. (WMAP Collaboration), Astrophys. J. Suppl. Ser. 208, 20 (2013).

[57] E. Aprile et al. (XENON Collaboration), Phys. Rev. Lett. 119, 181301 (2017).

[58] E. Aprile et al. (XENON Collaboration), J. Cosmol. Astropart. Phys. 04 (2016) 027.

[59] The ATLAS Collaboration, Report No. ATLAS-CONF2017-039.

[60] CMS Collaboration, Report No. CMS-PAS-SUS-16-039.

[61] H. Baer, A. Mustafayev, and X. Tata, Phys. Rev. D 90, 115007 (2014).

[62] C. Han, A. Kobakhidze, N. Liu, A. Saavedra, L. Wu, and J. M. Yang, J. High Energy Phys. 02 (2014) 049.

[63] The ATLAS collaboration, ATLAS-CONF-2016-096.

[64] M. Aaboud et al. (ATLAS Collaboration), Eur. Phys. J. C 78, 154 (2018).

[65] M. Drees, H. Dreiner, D. Schmeier, J. Tattersall, and J. S. Kim, Comput. Phys. Commun. 187, 227 (2015). 
[66] J. S. Kim, D. Schmeier, J. Tattersall, and K. Rolbiecki, Comput. Phys. Commun. 196, 535 (2015).

[67] D. Dercks, N. Desai, J. S. Kim, K. Rolbiecki, J. Tattersall, and T. Weber, Comput. Phys. Commun. 221, 383 (2017).

[68] T. Sjstrand, S. Ask, J. R. Christiansen, R. Corke, N. Desai, P. Ilten, S. Mrenna, S. Prestel, C. O. Rasmussen, and P.Z. Skands, Comput. Phys. Commun. 191, 159 (2015).

[69] J. de Favereau, C. Delaere, P. Demin, A. Giammanco, V. Lemaître, A. Mertens, and M. Selvaggi (DELPHES 3 Collaboration), J. High Energy Phys. 02 (2014) 057.
[70] W. Beenakker, R. Hopker, and M. Spira, arXiv:hep-ph/ 9611232

[71] J. Alwall, R. Frederix, S. Frixione, V. Hirschi, F. Maltoni, O. Mattelaer, H.-S. Shao, T. Stelzer, P. Torrielli, and M. Zaro, J. High Energy Phys. 07 (2014) 079.

[72] T. Sjostrand, S. Mrenna, and P. Z. Skands, J. High Energy Phys. 05 (2006) 026.

[73] M. Cacciari, G. P. Salam, and G. Soyez, J. High Energy Phys. 04 (2008) 063.

[74] Q. F. Xiang, X. J. Bi, P. F. Yin, and Z. H. Yu, Phys. Rev. D 94, 055031 (2016). 